\title{
Predictors for Disease-Modifying Anti-Rheumatic Drugs in Rheumatoid Arthritis Patients: A Cross-sectional Study
}

\section{Tommy T Cheung, Man Fung Tsoi, Bernard My Cheung}

Department of Medicine, The University of Hong Kong, Hong Kong

Introduction: Compliance of disease modifying anti-rheumatic drugs (DMARDs) could affect the effectiveness of DMARDs for the treatment of rheumatoid arthritis in real life clinical setting. Factors affecting compliance vary in different population and health care systems. Therefore, we identified the factors contributing to lower degree of compliance to DMARDs.

Method: This study was conducted in Queen Mary Hospital. For inclusion, rheumatoid arthritis patients must be treated with at least one $\operatorname{DMARD}(\mathrm{s})$. Levels of compliance and beliefs about medications were assessed using 19-item Compliance Questionnaire Rheumatology and the Beliefs about Medicine Questionnaire (BMQ) respectively. Demographic variables, disease activities and health states were collected during patient visits. Results were analysed by using SPSS v25.0. Logistic regression was used to identify the predictors for lower degree of compliance.

Results: 436 patients were recruited into this study. Female sex and young age (56.8 \pm 0.60 vs. $60.4 \pm 1.69 ; p=0.031)$ were associated with low levels of compliance. Results for logistic regression are summarised in table. Lower BMQ necessity score and higher BMQ concerns score were associated with low level of compliance. There was no significant difference in terms of educational levels, disease duration and activities, and, number of DMARDs used as stratified by the level of compliance.

Conclusion: Low level of compliance was associated with lower level of necessity and higher level of concerns about DMARDs. 